\title{
Psychiatric diagnoses in older people with intellectual disability in comparison with the general population: a register study
}

\author{
A. Axmon ${ }^{1 *}$, P. Björne ${ }^{2}$, L. Nylander ${ }^{3,4}$ and G. Ahlström ${ }^{5}$ \\ ${ }^{1}$ Department of Occupational and Environmental Medicine, Lund University, SE-221 00 Lund, Sweden \\ ${ }^{2}$ Research and Development Unit, City Office, City of Malmö, Sweden \\ ${ }^{3}$ Department of Clinical Sciences/Psychiatry, Lund University, Lund, Sweden \\ ${ }^{4}$ Gillberg Neuropsychiatry Centre, University of Gothenburg, Göteborg, Sweden \\ ${ }^{5}$ Department of Health Sciences, Lund University, Lund, Sweden
}

Aims. To describe the occurrence of psychiatric diagnoses in a specialist care setting in older people with intellectual disability (ID) in relation to those found in the same age group in the general population.

Method. A cohort of people with ID ( $n=7936)$, aged 55 years or more in 2012, was identified, as was an age and sex-matched cohort from the general population $(n=7936)$. Information regarding psychiatric diagnoses during 2002-2012 was collected from the National Patient Register, which contains records from all inpatient care episodes and outpatient specialist visits in Sweden. The mean age at the start of data collection (i.e. January 1st, 2002) was 53 years (range $44-85$ years).

Results. Seventeen per cent $(n=1382)$ of the people in the ID cohort had at least one psychiatric diagnosis recorded during the study period. The corresponding number in the general population cohort was $10 \%(n=817)$, which translates to an odds ratio (OR) of 1.84. The diagnoses recorded for the largest number of people in the ID cohort were 'other' (i.e. not included in any of the diagnostic groups) psychiatric diagnoses ( $10 \%$ of the cohort had at least one such diagnosis recorded) and affective disorders ( $7 \%$ ). In the general population cohort, the most common diagnoses were affective disorders $(4 \%)$ and alcohol/substance-abuse-related disorders (4\%). An increased odds of having at least one diagnosis was found for all investigated diagnoses except for alcohol/substance-abuse-related disorders $(\mathrm{OR}=0.56)$. The highest odds for the ID cohort was found for diagnosis of psychotic disorder ( $\mathrm{OR}=10.4)$ followed by attention deficit/hyperactive disorder $(\mathrm{OR}=3.81)$, dementia $(\mathrm{OR}=2.71)$, personality disorder $(\mathrm{OR}=2.67)$, affective disorder $(\mathrm{OR}=$ $1.74)$ and anxiety disorder $(\mathrm{OR}=1.36)$. People with ID also had an increased odds of psychiatric diagnoses not included in any of these groups $(\mathrm{OR}=8.02)$. The percentage of people with ID who had at least one diagnosis recorded during the study period decreased from more than 30\% among those aged 55-59 years in 2012 (i.e. born 1953-1957) to approximately $20 \%$ among those aged $75+$ years in 2012 (i.e. born in or before 1937).

Conclusions. Older people with ID seem to be more likely to have psychiatric diagnoses in inpatient or outpatient specialist care than their peers in the general population. If this is an effect of different disorder prevalence, diagnostic difficulties or differences in health care availability remains unknown. More research is needed to understand the diagnostic and treatment challenges of psychiatric disorders in this vulnerable group.

Received 28 September 2016; Accepted 27 January 2017; First published online 23 February 2017

Key words: Diagnosis, elderly, mental health, mental retardation.

\section{Introduction}

The number of people with intellectual disability (ID) who reach older age is increasing (Haveman, 2004; Coppus, 2013; Dieckmann et al. 2015). Moreover, the ageing process starts at a younger age among people with ID (WHO, 2000), and they show signs of frailty earlier than

* Address for correspondence: Dr A. Axmon, Department of Occupational and Environmental Medicine, Lund University, SE-221 00 Lund, Sweden.

(Email: anna.axmon@med.lu.se) the general population (Evenhuis et al. 2012). A few studies that have focused on psychiatric diagnoses among older people with ID suggest that old age increases the risk of overall psychiatric morbidity, dementia, anxiety disorder and depression (Cooper, 1997a, b; Deb et al. 2001). In wider and/or younger age groups, people with ID may have a higher risk for psychiatric disorders than the general population (Bhaumik et al. 2008; Nettelbladt et al. 2009; Yoo et al. 2012). If this can be extrapolated into older age groups is uncertain.

An accurate psychiatric diagnosis for a person with ID can be difficult to make, due to e.g. communication

This is an Open Access article, distributed under the terms of the Creative Commons Attribution licence (http://creativecommons.org/licenses/by/4.0/), which permits unrestricted re-use, distribution, and reproduction in any medium, provided the original work is properly cited. 
difficulties, physical health issues (Bhaumik et al. 2008), diagnostic overshadowing (Reiss \& Szyszko, 1983) and lack of assessment tools adapted for people with ID (Alexander \& Cooray, 2003; Moreland et al. 2008; Hermans \& Evenhuis, 2010). This is most likely true for all psychiatric diagnoses for which there are no objective markers, i.e. with disorders due to alcohol or substance use as the only exception. Due to this, a large part of psychiatric morbidity in people with ID may be hidden (Salvador-Carulla et al. 2000), and it has been suggested that people with ID do not receive the services that their health conditions require (Ouellette-Kuntz et al. 2005).

Having one psychiatric disorder seems to be a risk factor for more psychiatric disorders among people with ID (Goldberg et al. 1995; Lidher et al. 2005; Bakken et al. 2010). Some psychiatric disorders have also been suggested to be associated with higher risk of physical disorders (e.g. Moss \& Patel, 1997; Cooper, 1999). Further, psychiatric disorders such as schizophrenia-spectrum psychoses appear to be more debilitating among people with ID (Bouras et al. 2004). Thus, failure to correctly diagnose psychiatric disorders in people with ID may have severe consequences for the individual.

By assessing patterns of psychiatric diagnoses in older people with ID, and comparing these to those in the general population, important knowledge can be made available to policy makers, health organizations and service providers. This knowledge may then provide the basis for improvements in support and service for ageing people with ID.

The aim of the present study was to describe the occurrence of psychiatric diagnoses in a specialist care setting in older people with ID in relation to those found in the same age group in the general population.

\section{Material and methods}

This was a register-based study investigating psychiatric diagnoses among older people with ID in comparison with same-aged people from the general population. The study cohorts were defined using national registers, and outcome data were collected from national registers.

\section{Study population}

In Sweden, people with an ID or autism spectrum disorder (ASD) can apply for services according to the Act Concerning Support and Service for People with Certain Functional Impairments (Swedish abbreviation: LSS) (SFS 1993:387, 1993). People with a diagnosis of ID or ASD are eligible to apply for support through a case manager in the municipality. All support provided by the municipality is documented in the LSS-register, which is managed by the Swedish National Board of Health and Welfare, a government agency under the Ministry of Health and Social Affairs.

In the present study, we used LSS support as a proxy for ID. Through the LSS-register, all people with at least one form of support, aged 55 years and above in 2012 were identified (ID cohort). In addition, they had to be alive at the end of that year. A one-to-one sex and age-matched control cohort from the general population (gPop cohort) was established by Statistics Sweden using the Swedish population register. People included in the ID cohort could not be included in the gPop cohort also. However, people with ID but without LSS support were not excluded from the gPop cohort.

\section{Outcomes}

The Swedish National Patient Register (NPR) is also managed by the Swedish National Board of Health and Welfare. It contains information about all in- and outpatient specialist care in Sweden. However, it does not contain information about visits to primary health care. For inpatient care, registration in the NPR is made at the date of discharge, and for outpatient care it is made at the date of the visit. For each registration, one primary and up to 21 secondary diagnoses are listed, coded according to the 10th revision of the International Classification of Disease (ICD-10).

Information on psychiatric diagnoses during the study period was obtained from the NPR for 20022012. The mean age at the start of data collection (i.e. 1 January 2002) was 53 years (range 44-85 years). These were categorised as attention deficit/hyperactivity disorder (ADHD) and equivalents, psychotic disorders, affective disorders, anxiety disorders, personality disorders, alcohol/substance-abuse-related disorders, dementia or other psychiatric disorders (Table 1). Each person was categorised as having none or at least one diagnosis in each diagnostic category during the study period, and the date of the first record of each diagnosis for each person was noted. We did not differentiate between primary and secondary diagnoses. Thus, a secondary diagnosis of e.g. dementia would classify an individual as having at least one dementia diagnosis even if the primary diagnosis for that visit was another psychiatric diagnosis.

\section{Statistics}

To compare the number of people with at least one of each respective category of psychiatric diagnoses in the 
Table 1. Diagnostic category definition and number of people in a general population sample (gPop, $n=7936)$ and among people with intellectual disability (ID, $n=7936)$ with at least one diagnosis and at least one primary diagnosis, respectively, during 2002-2012

\begin{tabular}{|c|c|c|c|c|}
\hline & \multicolumn{2}{|c|}{ gPop } & \multicolumn{2}{|c|}{ ID } \\
\hline & $\begin{array}{c}\text { All } \\
n(\%)\end{array}$ & $\begin{array}{c}\text { Primary } \\
n(\%)\end{array}$ & $\begin{array}{c}\text { All } \\
n(\%)\end{array}$ & $\begin{array}{c}\text { Primary } \\
n(\%)\end{array}$ \\
\hline \multicolumn{5}{|l|}{ Attention deficit/hyperactivity disorder (ADHD) and equivalents } \\
\hline Hyperkinetic disorders (F90) & $10(0.1)$ & $5(0.1)$ & $38(0.5)$ & $18(0.2)$ \\
\hline \multicolumn{5}{|l|}{ Psychotic disorders } \\
\hline Schizophrenia (F20) & $16(0.2)$ & $12(0.2)$ & $210(2.6)$ & $120(1.5)$ \\
\hline Schizotypal disorder (F21) & $<5$ & $<5$ & $5(0.1)$ & $<5$ \\
\hline Persistent delusional disorders (F22) & $9(0.1)$ & $7(0.1)$ & $63(0.8)$ & $38(0.5)$ \\
\hline Acute and transient psychotic disorders (F23) & $6(0.1)$ & $<5$ & $51(0.6)$ & $25(0.3)$ \\
\hline Induced delusional disorder (F24) & $<5$ & $<5$ & $<5$ & $<5$ \\
\hline Schizoaffective disorders (F25) & $6(0.1)$ & $<5$ & $46(0.6)$ & $30(0.4)$ \\
\hline Other nonorganic psychotic disorders (F28) & $<5$ & $<5$ & $12(0.2)$ & $<5$ \\
\hline Unspecified nonorganic psychosis (F29) & $9(0.1)$ & $<5$ & $128(1.6)$ & $60(0.8)$ \\
\hline \multicolumn{5}{|l|}{ Affective disorders } \\
\hline Manic episode (F30) & $<5$ & $<5$ & $32(0.4)$ & $19(0.2)$ \\
\hline Bipolar affective disorder (F31) & $42(0.5)$ & $37(0.5)$ & $170(2.1)$ & $125(1.6)$ \\
\hline Depressive episode (F32) & $254(3.2)$ & $182(2.3)$ & $358(4.5)$ & $208(2.6)$ \\
\hline Recurrent depressive disorder (F33) & $116(1.5)$ & $93(1.2)$ & $116(1.5)$ & $83(1.0)$ \\
\hline Persistent mood [affective] disorders (F34) & $23(0.3)$ & $14(0.2)$ & $21(0.3)$ & $11(0.1)$ \\
\hline Other mood [affective] disorders (F38) & $5(0.1)$ & $<5$ & $<5$ & $<5$ \\
\hline Unspecified mood [affective] disorder (F39) & $17(0.2)$ & $10(0.1)$ & $35(0.4)$ & $17(0.2)$ \\
\hline \multicolumn{5}{|l|}{ Anxiety disorders } \\
\hline Phobic anxiety disorders (F40) & $16(0.2)$ & $10(0.1)$ & $36(0.5)$ & $18(0.2)$ \\
\hline Other anxiety disorders (F41) & $196(2.5)$ & $131(1.7)$ & $289(3.6)$ & $219(2.8)$ \\
\hline Obsessive-compulsive disorder (F42) & $10(0.1)$ & $6(0.1)$ & $74(0.9)$ & $34(0.4)$ \\
\hline Reaction to severe stress and adjustment disorders (F43) & $143(1.8)$ & $116(1.5)$ & $81(1.0)$ & $66(0.8)$ \\
\hline \multicolumn{5}{|l|}{ Personality disorders } \\
\hline Specific personality disorders (F60) & $22(0.3)$ & $13(0.2)$ & $60(0.8)$ & $30(0.4)$ \\
\hline Mixed and other personality disorders (F61) & $<5$ & $<5$ & $<5$ & $<5$ \\
\hline \multicolumn{5}{|l|}{ Alcohol/substance-abuse-related disorders } \\
\hline \multicolumn{5}{|l|}{ Mental and behavioural disorders due to use of } \\
\hline opioids (F11) & $23(0.3)$ & $14(0.2)$ & $8(0.1)$ & $7(0.1)$ \\
\hline cannabinoids (F12) & $7(0.1)$ & $<5$ & $6(0.1)$ & $<5$ \\
\hline sedatives or hypnotics (F13) & $36(0.5)$ & $17(0.2)$ & $8(0.1)$ & $5(0.1)$ \\
\hline cocaine $(\mathrm{F} 14)$ & $<5$ & $<5$ & $<5$ & $<5$ \\
\hline other stimulants, including caffeine (F15) & $11(0.1)$ & $7(0.1)$ & $<5$ & $<5$ \\
\hline hallucinogens (F16) & $1(<0.1)$ & $<5$ & $<5$ & $<5$ \\
\hline tobacco $(\mathrm{F} 17)$ & $87(1.1)$ & $6(0.1)$ & $43(0.5)$ & $6(0.1)$ \\
\hline volatile solvents $(\mathrm{F} 18)$ & $<5$ & $<5$ & $<5$ & $<5$ \\
\hline multiple drug use and use of other psychoactive substances (F19) & $33(0.4)$ & $21(0.3)$ & $19(0.2)$ & $11(0.1)$ \\
\hline \multicolumn{5}{|l|}{ Dementia } \\
\hline Dementia in Alzheimer disease (F00) & $24(0.3)$ & $15(0.2)$ & $51(0.6)$ & $26(0.3)$ \\
\hline Vascular dementia (F01) & $18(0.2)$ & $11(0.1)$ & $22(0.3)$ & $<5$ \\
\hline Dementia in other diseases classified elsewhere (F02) & $<5$ & $<5$ & $8(0.1)$ & $<5$ \\
\hline Unspecified dementia (F03) & $38(0.5)$ & $15(0.2)$ & $133(1.7)$ & $33(0.4)$ \\
\hline Delirium superimposed on dementia (F05.1) & $<5$ & $<5$ & $<5$ & $<5$ \\
\hline Alcoholic dementia (F10.7A) & $<5$ & $<5$ & $<5$ & $<5$ \\
\hline Alzheimer disease (G30) & $31(0.4)$ & $22(0.3)$ & $60(0.8)$ & $23(0.3)$ \\
\hline $\begin{array}{l}\text { Other degenerative diseases of nervous system, not } \\
\text { elsewhere classified (G31) }\end{array}$ & $10(0.1)$ & $7(0.1)$ & $11(0.1)$ & $<5$ \\
\hline
\end{tabular}


Table 1. Continued

\begin{tabular}{|c|c|c|c|c|}
\hline & \multicolumn{2}{|c|}{ gPop } & \multicolumn{2}{|c|}{ ID } \\
\hline & $\begin{array}{c}\text { All } \\
n(\%)\end{array}$ & $\begin{array}{l}\text { Primary } \\
n(\%)\end{array}$ & $\begin{array}{c}\text { All } \\
n(\%)\end{array}$ & $\begin{array}{c}\text { Primary } \\
n(\%)\end{array}$ \\
\hline \multicolumn{5}{|l|}{ Other psychiatric disorders } \\
\hline Delirium, not induced by alcohol and other psychoactive substances (F05) & $9(0.1)$ & $5(0.1)$ & $14(0.2)$ & $11(0.1)$ \\
\hline $\begin{array}{l}\text { Other mental disorders due to brain damage and dysfunction and to physical } \\
\text { disease (F06) }\end{array}$ & $21(0.3)$ & $9(0.1)$ & $132(1.7)$ & $45(0.6)$ \\
\hline $\begin{array}{l}\text { Personality and behavioural disorders due to brain disease, damage and } \\
\text { dysfunction (F07) }\end{array}$ & $6(0.1)$ & $<5$ & $39(0.5)$ & $17(0.2)$ \\
\hline Unspecified organic or symptomatic mental disorder (F09) & $<5$ & $<5$ & $24(0.3)$ & $9(0.1)$ \\
\hline Dissociative [conversion] disorders (F44) & $<5$ & $<5$ & $27(0.3)$ & $18(0.2)$ \\
\hline Somatoform disorders (F45) & $48(0.6)$ & $34(0.4)$ & $31(0.4)$ & $22(0.3)$ \\
\hline Other neurotic disorders (F48) & $5(0.1)$ & $<5$ & $11(0.1)$ & $6(0.1)$ \\
\hline Eating disorders (F50) & $<5$ & $<5$ & $7(0.1)$ & $<5$ \\
\hline Nonorganic sleep disorders (F51) & $16(0.2)$ & $4(0.1)$ & $10(0.1)$ & $<5(<0.1)$ \\
\hline Sexual dysfunction, not caused by organic disorder or disease (F52) & $<5$ & $<5$ & $<5$ & $<5$ \\
\hline $\begin{array}{l}\text { Mental and behavioural disorders associated with the puerperium, not elsewhere } \\
\text { classified (F53) }\end{array}$ & $<5$ & $<5$ & $<5$ & $<5$ \\
\hline Habit and impulse disorders (F63) & $<5$ & $<5$ & $10(0.1)$ & $<5$ \\
\hline Disorders of sexual preference (F65) & $<5$ & $<5$ & $5(0.1)$ & $<5$ \\
\hline Other disorders of adult personality and behaviour (F68) & $<5$ & $<5$ & $<5$ & $<5$ \\
\hline Unspecified disorder of adult personality and behaviour (F69) & $<5$ & $<5$ & $10(0.1)$ & $<5$ \\
\hline Specific developmental disorders of speech and language (F80) & $<5$ & $<5$ & $68(0.9)$ & $<5$ \\
\hline Specific developmental disorders of scholastic skills (F81) & $<5$ & $<5$ & $11(0.1)$ & $<5$ \\
\hline Mixed specific developmental disorders (F83) & $<5$ & $<5$ & $<5$ & $<5$ \\
\hline Unspecified disorder of psychological development (F89) & $<5$ & $<5$ & $395(5.0)$ & $96(1.2)$ \\
\hline Conduct disorders (F91) & $<5$ & $<5$ & $13(0.2)$ & $6(0.1)$ \\
\hline Mixed disorders of conduct and emotions (F92) & $<5$ & $<5$ & $<5$ & $<5$ \\
\hline Emotional disorders with onset specific to childhood (F93) & $<5$ & $<5$ & $<5$ & $<5$ \\
\hline $\begin{array}{l}\text { Disorders of social functioning with onset specific to childhood and } \\
\text { adolescence (F94) }\end{array}$ & $<5$ & $<5$ & $<5$ & $<5$ \\
\hline Tic disorders (F95) & $<5$ & $<5$ & $16(0.2)$ & $<5$ \\
\hline $\begin{array}{l}\text { Other behavioural and emotional disorders with onset usually occurring in } \\
\text { childhood and adolescence (F98) }\end{array}$ & $<5$ & $<5$ & $23(0.3)$ & $<5$ \\
\hline Mental disorder, not otherwise specified (F99) & $<5$ & $<5$ & $31(0.4)$ & $<5$ \\
\hline Auditory hallucinations (R44.0) & $<5$ & $<5$ & $12(0.2)$ & $<5$ \\
\hline
\end{tabular}

Numbers are not given for cells where there are less than five observations.

ID cohort to the corresponding number in the gPop cohort, we estimated odds ratios (ORs) with 95\% confidence intervals (CIs) using logistic regression. In order to illustrate possible age-effects, we performed age stratified analyses, using the 5-year age categories. Moreover, we investigated age effects within the ID cohort by comparing each age group to the youngest one. Statistical interaction was evaluated by introducing an interaction term (e.g. cohort*age group) to the logistic regression model, and trends were assessed by treating the category variable as a continuous factor.

In order to evaluate using the LSS register as a proxy for ID, we performed sensitivity analyses on subcohorts of people with known diagnosis of either ID or ASD. Through diagnoses available from the NPR, we were able to identify 1145 men and 1002 women who had at least one diagnosis of ID (F7 in ICD-10) during 2002-2012. Moreover, we identified 242 men and 156 women who had at least one diagnosis of ASD (F84, excluding F84.1 and F84.5, in ICD-10). The overlap was 209 individuals. Analyses were made comparing psychiatric diagnoses among those with ASD only to those with ID only or ASD in combination with ID. Also, as people with ID may be difficult to diagnose with respect to psychiatric disorders, we performed sensitivity analyses including only diagnoses recorded at psychiatric clinics, i.e. by psychiatric specialists.

Analyses were only performed if each of the two compared group comprised at least five observations. 
A two-tailed $p$-value of 0.05 was considered statistically significant. All analyses were performed in IBM SPSS Statistics 23.

\section{Results}

Each cohort comprised 7936 people, whereof $45 \%$ were women. The percentage of women increased over the age categories, with $43 \%$ among those aged $55-59$ years (total $n=2559$ in each cohort), 44\% among those aged 60-64 years $(n=2097), 46 \%$ among those aged 65-69 years $(n=1636), 48 \%$ among those aged $70-74$ years $(n=839)$ and $53 \%$ among those aged $75+$ years $(n=805)$.

Figure 1 describes the cumulative number of people with a recorded diagnosis during the study period, by cohort, age group and diagnostic category. The rightmost end of each line corresponds to the number of people for whom each diagnosis was recorded at least once during the study period, which is also given in Table 2. Increased ORs for the ID cohort were found for all diagnostic categories except alcohol/substance-abuse-related disorders. The ORs that indicated increased odds for the ID cohort ranged from 1.36 (anxiety disorders) to 10.4 (psychotic disorders). Sensitivity analyses including only diagnoses recorded in psychiatric care increased the OR for dementia by $60 \%$ (from 2.71 to 4.34 ), for 'other psychiatric diagnosis' by $29 \%$ (from 8.02 to 10.32 ), for any psychiatric diagnosis by $24 \%$ (from 1.84 to 2.28 ), and for anxiety disorders by $16 \%$ (from 1.36 to 1.58 ). For all other diagnostic categories, the results remained similar (less than 15\% change; ORs not shown).

When stratifying by age group, the results were consistent with those found for the whole cohorts for ADHD as well as psychotic, affective, anxiety, personality and 'other' psychiatric disorders. However, for alcohol/substance-abuse-related disorders, the OR for the ID cohort $v$. the gPop cohort decreased, such that it moved away from the null, with increasing age. Moreover, for dementia and any psychiatric disorder, the OR for the ID cohort $v$. the gPop cohort decreased towards the null with age.

Within the ID cohort, the oldest age group had lower odds than the youngest age group of psychotic, affective, and anxiety disorders, as well as of dementia and any psychiatric diagnosis (Table 3). No such effect was found for 'other' psychiatric diagnoses. We could not perform the corresponding analyses for ADHD, personality disorders, or alcohol/substanceabuse-related disorders as the number with recorded diagnoses in the oldest age group was too low. However, the results from the two middle age groups suggested that the odds of diagnoses of ADHD or alcohol/substance-abuse-related disorders also increased with age. In the sensitivity analyses, those with ASD only had lower odds of receiving psychiatric diagnoses than those with ID only or those with ID in combination with ASD, with exception of diagnoses of ADHD (Table 4). However, statistical significance was achieved only for affective disorders, 'other psychiatric diagnoses' (only in comparison with ID and ASD in combination), and 'any' psychiatric diagnosis.

\section{Discussion}

Older people with ID had higher odds than their counterparts in the general population to have at least one psychiatric diagnosis recorded in inpatient or outpatient specialist care. The largest discrepancy between the two cohorts was for psychotic disorders, of which people with ID had more than ten times the odds of having a diagnosis recorded during the 11-year study period. The only diagnostic category for which a higher odds was found in the general population was alcohol/substance-abuse-related disorders.

The ID cohort is an administratively identified group of people with ID in Sweden, using the LSS-register. The people in this group were 55 years and older in 2012, and thereby belong to the group of people with ID who received services before the LSS Act (SFS 1993:387, 1993) was passed in 1993. Prior to this, people who had a diagnosis of ID were more or less automatically registered for service based on their diagnosis, and not as in the current act, after applying for support. Therefore, the cohort can be expected to fairly well cover the group of ageing people with ID in Sweden. Nevertheless, the register does not contain information on the diagnosis that was the basis for the provision of services (i.e. ID or ASD). Thereby we cannot distinguish those who have ID only or ID in combination with ASD from those who have ASD only. Sensitivity analyses showed that those with ASD only had lower odds than those with ID only or ID in combination with ASD to have different psychiatric diagnoses recorded in the NPR. Thus, the higher the fraction of people with ASD only in the ID cohort, the more likely we would be to underestimate the effect of having ID.

It is important not to confuse psychiatric diagnoses with psychiatric disorders. Whereas a disorder relates to the actual state of health of a person, a diagnosis is merely a proxy thereof. If it is more likely that a disorder will go undiagnosed than it is that a diagnosis is not linked to a disorder in a person, the number of diagnoses will tend to underestimate the true numbers, especially in non-chronic disorders. The opposite is true if diagnoses are given without an underlying disorder, e.g. if a behaviour resulting from environmental stressors is misinterpreted as symptoms of a psychiatric disorder. 


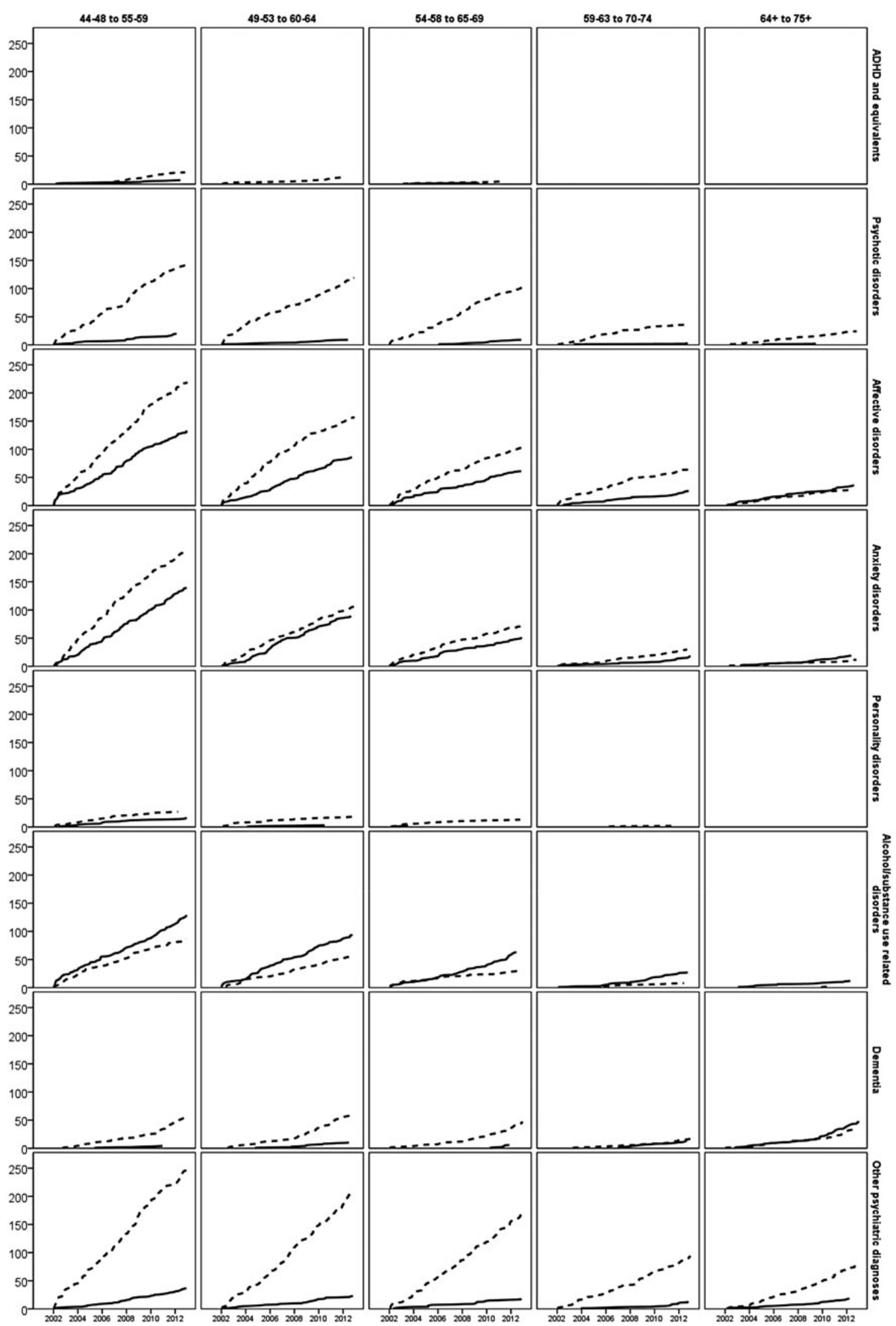

Fig. 1. Cumulative number of people with diagnoses within different diagnostic categories during 2002-2012 in a cohort of 7936 people with ID (broken lines) and a same-sized sample from the general population, one-to-one matched by age and sex (gPop, solid lines). 
Table 2. Number of people with at least one diagnosis in different psychiatric diagnostic categories during 2002-2012, among people with intellectual disability (ID) and a one-to-one age-and sex-matched sample from the general population $(\mathrm{gPop})$

\begin{tabular}{|c|c|c|c|c|c|c|c|c|c|}
\hline & $\mathrm{ADHD}^{\mathrm{a}}$ & $\begin{array}{l}\text { Psychotic } \\
\text { disorders }\end{array}$ & $\begin{array}{l}\text { Affective } \\
\text { disorders }\end{array}$ & $\begin{array}{l}\text { Anxiety } \\
\text { disorders }\end{array}$ & $\begin{array}{l}\text { Personality } \\
\text { disorders }\end{array}$ & $\begin{array}{c}\text { Alcohol/ } \\
\text { substance-abuse-rel. } \\
\text { disorders }\end{array}$ & Dementia & $\begin{array}{l}\text { Other psychiatric } \\
\text { disorders }\end{array}$ & $\begin{array}{c}\text { Any psychiatric } \\
\text { disorder }\end{array}$ \\
\hline \multicolumn{10}{|c|}{ All $(n=7936$ in each cohort $)$} \\
\hline gPop, $n(\%)$ & $1<5$ & $43(1)$ & $342(4)$ & $317(4)$ & $23(0)$ & $325(4)$ & $81(1)$ & $109(1)$ & $817(10)$ \\
\hline ID, $n(\%)$ & $38(0)$ & $425(5)$ & $576(7)$ & $425(5)$ & $61(1)$ & $186(2)$ & $216(3)$ & $797(10)$ & $1382(17)$ \\
\hline ID v. gPop & $3.81(1.90-7.66)$ & $10.4(7.58-14.2)$ & $1.74(1.51-1.99)$ & $1.36(1.17-1.58)$ & $2.66(1.65-4.31)$ & $0.56(0.47-0.67)$ & $2.71(2.10-3.51)^{\mathrm{b}}$ & $8.02(6.55-9.82)$ & $1.84(1.68-2.02)$ \\
\hline Specialist $^{\mathrm{c}}$ & $5.35(2.24-12.8)$ & $11.9(8.05-17.7)$ & $1.66(1.43-1.93)$ & $1.33(1.14-1.57)$ & $2.25(1.34-3.76)$ & $0.60(0.48-0.75)$ & $2.36(1.72-3.24)$ & $5.28(4.15-6.72)$ & $1.84(1.66-2.04)$ \\
\hline \multicolumn{10}{|c|}{$44-48$ in 2002 to $55-59$ in 2012 ( $n=2559$ in each cohort) } \\
\hline gPop, $n(\%)$ & $7(0)$ & $20(1)$ & $133(5)$ & $140(5)$ & $17(1)$ & $129(5)$ & $<5$ & $37(1)$ & $291(11)$ \\
\hline $\mathrm{ID}, n(\%)$ & $21(1)$ & $143(6)$ & $219(9)$ & $205(8)$ & $27(1)$ & 87 (3) & $56(2)$ & $247(10)$ & $526(21)$ \\
\hline ID $v \cdot$ gPop & $3.02(1.28-7.11)$ & $7.51(4.69-12.0)$ & $1.71(1.37-2.13)$ & $1.50(1.20-1.88)$ & $1.59(0.87-2.93)$ & $0.66(0.50-0.88)$ & $\mathrm{NC}$ & $7.28(5.13-10.3)$ & $2.02(1.73-2.36)$ \\
\hline \multicolumn{10}{|c|}{$49-53$ in 2002 to $60-64$ in 2012 ( $n=2097$ in each cohort) } \\
\hline gPop, $n(\%)$ & $<5$ & $9(0)$ & $86(4)$ & $89(4)$ & $<5$ & $94(4)$ & $1<5$ & $23(1)$ & $213(10)$ \\
\hline $\mathrm{ID}, n(\%)$ & $12(1)$ & $119(6)$ & $157(7)$ & $106(5)$ & $18(1)$ & $56(3)$ & $60(3)$ & $209(10)$ & $374(18)$ \\
\hline ID $v$. gPop & $\mathrm{NC}$ & $14.0(7.07-27.6)$ & $1.89(1.44-2.48)$ & $1.20(0.90-1.60)$ & NC & $0.58(0.42-0.82)$ & $6.15(3.14-12.0)$ & $9.98(6.46-15.4)$ & $1.92(1.60-2.30)$ \\
\hline \multicolumn{10}{|c|}{$54-58$ in 2002 to $65-69$ in 2012 ( $n=1636$ in each cohort) } \\
\hline gPop, $n(\%)$ & $<5$ & $9(1)$ & $61(4)$ & $51(3)$ & $<5$ & $63(4)$ & $6(0)$ & $18(1)$ & $141(9)$ \\
\hline ID, $n(\%)$ & $5(0)$ & $102(6)$ & $105(6)$ & $72(4)$ & $13(1)$ & $32(2)$ & $47(3)$ & $170(10)$ & $272(17)$ \\
\hline ID v. gPop & $2.50(0.49-12.9)$ & $12.0(6.06-23.8)$ & $1.77(1.28-2.45)$ & $1.43(0.99-2.06)$ & $\mathrm{NC}$ & $0.50(0.32-0.77)$ & $8.04(3.43-18.8)$ & $10.4(6.38-17.0)$ & $2.11(1.70-2.62)$ \\
\hline \multicolumn{10}{|c|}{$59-63$ in 2002 to $70-74$ in 2012 ( $n=839$ in each cohort) } \\
\hline gPop, $n(\%)$ & $<5$ & $<5$ & $26(3)$ & $18(2)$ & $<5$ & $27(3)$ & $13(2)$ & $12(1)$ & $77(9)$ \\
\hline ID, $n(\%)$ & $<5$ & $37(4)$ & $67(8)$ & $30(4)$ & $<5$ & $8(1)$ & $17(2)$ & $94(11)$ & $123(15)$ \\
\hline ID v. gPop & $\mathrm{NC}$ & $\mathrm{NC}$ & $2.71(1.71-4.31)$ & $1.69(0.94-3.06)$ & NC & $0.29(0.13-0.64)$ & $1.31(0.63-2.72)$ & $8.70(4.73-16.0)$ & $1.70(1.26-2.30)$ \\
\hline \multicolumn{10}{|c|}{$64+$ in 2002 to $75+$ in 2012 ( $n=805$ in each cohort) } \\
\hline gPop, $n(\%)$ & $<5$ & $<5$ & $36(4)$ & $19(2)$ & $<5$ & $12(1)$ & $48(6)$ & $19(2)$ & $95(12)$ \\
\hline $\mathrm{ID}, n(\%)$ & $<5$ & $24(3)$ & $28(3)$ & $12(1)$ & $<5$ & $<5$ & $36(4)$ & $77(10)$ & $87(11)$ \\
\hline ID $v$. gPop & $\mathrm{NC}$ & $\mathrm{NC}$ & $0.77(0.47-1.27)$ & $0.63(0.30-1.30)$ & $\mathrm{NC}$ & NC & $0.74(0.47-1.15)$ & $4.38(2.62-7.30)$ & $0.91(0.67-1.23)$ \\
\hline$p_{\text {interaction }}$ & 0.696 & 0.277 & 0.318 & 0.198 & 0.107 & 0.026 & $<0.001$ & 0.310 & $<0.001$ \\
\hline
\end{tabular}

$\mathrm{NC}$, not calculated as at least one cell contains less than five observations.

Comparisons of ID $v$. gPop are done using logistic regression, thus estimating OR with $95 \%$ confidence intervals. $\mathrm{p}$ values relate to the possible interaction between age group and ID. Statistically significant results are marked in bold.

a Attention deficit/hyperactive disorder.

boriginally presented in Axmon et al. (2016).

'Subgroup analyses including only diagnoses recorded in specialist care. 


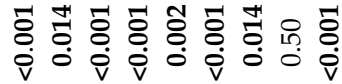
交金

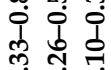

สิ ิิ ิิ)

i t

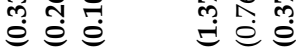

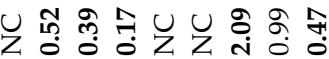

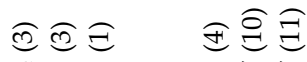

ம)

बิศ

辛定

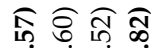

का ने

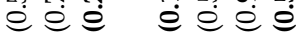

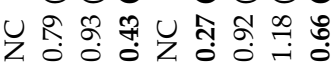

平包声

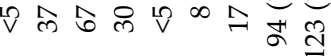

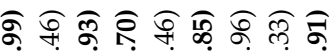

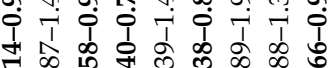

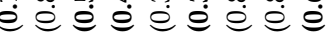

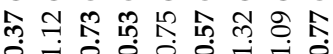

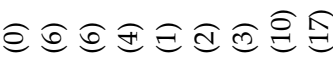

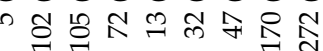

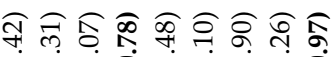

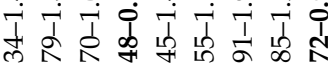
○े $0 \dot{0} 0$

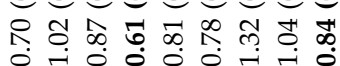

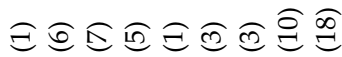

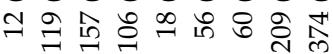

The numbers provided in this study are prevalences for the study period. For chronic disorders, these prevalences may be used as estimates of point prevalence at the study end, and may therefore be compared with point prevalences presented in other studies. However, for non-chronic disorders, such comparisons are not meaningful.

Diagnosing psychiatric disorders in people with ID may be difficult, as the patient must not only recognise the symptoms but also be able to communicate them, which is not always the case, especially among those with severe or profound ID. Diagnosing is further complicated by diagnostic overshadowing (Reiss \& Szyszko, 1983), i.e. when professionals attribute the symptoms of the psychiatric disorder to the ID, and masking, i.e. when the clinical characteristics of the psychiatric disorder are considered secondary to the ID. As a consequence of these diagnostic challenges, a large part of psychiatric morbidity in people with ID is hidden (Salvador-Carulla et al. 2000), which may lead to an undercount in health care among people with ID. For some psychiatric disorders, assessment tools are available, at least for people with mild or moderate ID (Mindham \& Espie, 2003; Deb et al. 2007; Antonacci \& Attiah, 2008; Perez-Achiaga et al. 2009; Havercamp \& Scott, 2015). For others, researchers have called for further development and evaluation of diagnostic systems for people with ID (Alexander \& Cooray, 2003; Moreland et al. 2008; Hermans \& Evenhuis, 2010). This is important to be aware of when comparing the occurrence of psychiatric diagnoses among people with ID to groups with more communicative skills, such as the general population.

There are different systems to classify psychiatric disorders among people with ID. Diagnoses in the Swedish NPR, on which the present study is based, are classified according to the ICD-10. However, diagnoses may also be made according to e.g. the Diagnostic and Statistical Manual of Mental Disorders (DSM) or the Diagnostic Criteria for psychiatric disorders for use with adults with Learning Disabilities/mental retardation (DC-LD). These are not identical with respect to diagnostic criteria, and so diagnoses may differ between them. Differences between diagnostic criteria have been found both in the general population (Erkinjuntti et al. 1997; Cheniaux et al. 2009; Nilsson et al. 2012) and among people with ID (Cooper et al. 2007b; Mantry et al. 2008; Melville et al. 2008; Strydom et al. 2008). If a diagnostic system is more or less likely to diagnose a person with ID than one from the general population, the potential risk of having the diagnosis associated with having ID would not be correctly estimated.

The training and experience of the professional making the diagnosis may also contribute to differences in diagnoses between groups. In order to 
Table 4. Number of people identified through the LSS register with at least one diagnosis in different psychiatric diagnostic categories in inpatient or specialist outpatient care during 2002-2012, grouped according to diagnosis of intellectual disability (ID, F7 in ICD-10) and autism spectrum disorder (ASD, F84, excluding F84.1 and F84.5, in ICD-10)

\begin{tabular}{|c|c|c|c|c|c|}
\hline & \multirow{2}{*}{$\begin{array}{l}\begin{array}{c}\text { ASD diagnosis } \\
\text { only }(n=189)\end{array} \\
n(\%)\end{array}$} & \multicolumn{2}{|c|}{$\begin{array}{l}\text { ID diagnosis only } \\
\qquad(n=1938)\end{array}$} & \multicolumn{2}{|c|}{$\begin{array}{l}\text { Both ID and ASD } \\
\text { diagnoses }(n=209)\end{array}$} \\
\hline & & $n(\%)$ & OR $(95 \%$ CI $)$ & $n(\%)$ & OR $(95 \%$ CI) \\
\hline Attention deficit/hyperactivity disorder (ADHD) & $3(2)$ & $12(1)$ & $0.39(0.11-1.38)$ & $3(1)$ & $0.90(0.18-4.53)$ \\
\hline Psychotic disorders & $19(10)$ & $293(15)$ & $1.59(0.98-2.60)$ & $24(11)$ & $1.16(0.61-2.19)$ \\
\hline Affective disorders & $20(11)$ & $332(17)$ & $1.75(1.08-2.82)$ & $41(20)$ & $2.06(1.16-3.67)$ \\
\hline Anxiety disorders & $14(7)$ & $213(11)$ & $1.54(0.88-2.71)$ & $28(13)$ & $1.93(0.99-3.80)$ \\
\hline Personality disorders & $2(1)$ & $27(1)$ & $1.32(0.31-5.60)$ & $2(1)$ & $0.90(0.13-6.48)$ \\
\hline Alcohol/substance-abuse-related disorders & $5(3)$ & $62(3)$ & $1.22(0.48-3.06)$ & $3(1)$ & $0.54(0.13-2.27)$ \\
\hline Dementia & $4(2)$ & $84(4)$ & $2.10(0.76-5.78)$ & $9(4)$ & $2.08(0.63-6.87)$ \\
\hline Other psychiatric diagnoses & $30(16)$ & $373(19)$ & $1.26(0.84-1.90)$ & $62(30)$ & $2.24(1.37-3.65)$ \\
\hline Any psychiatric diagnosis & $45(24)$ & $727(38)$ & $1.92(1.36-2.72)$ & $80(38)$ & $1.98(1.28-3.07)$ \\
\hline
\end{tabular}

Statistically significant results are marked in bold.

investigate whether this may be an issue in the present study, we performed sensitivity analyses including only diagnoses recorded in psychiatric care. In the sensitivity analyses, ORs associated with ID increased for dementia, anxiety disorders, 'other' psychiatric disorders and any psychiatric disorder. If psychiatric diagnoses made in psychiatric care are more correct than diagnoses recorded in somatic care, this implies that these disorders may go undiagnosed if the person with ID is treated only in somatic clinics.

Over the 11-year study period, people with ID had three times higher odds than those in the general population to have at least one psychiatric diagnosis recorded in inpatient or outpatient specialist care. Previous studies have investigated prevalence of psychiatric diagnoses among adults up to 64 years of age and with mild or moderate ID, using diagnoses according to the ICD-10 (Deb et al. 2001; Schutzwohl et al. 2016). When comparing these prevalences to those found in the general population, neither of them found an increased prevalence associated with ID. In studies including people older than 65 years and without restrictions regarding severity of ID, different patterns have been found. Cooper et al. (2007b) found a higher prevalence among people with ID than what was found in the general population in the UK when diagnosing according to the DC-LD, but not when using ICD-10 or DSM-IV. Nettelbladt et al. (2009) found a 34\% increased (based on cumulative incidence) for psychiatric diagnoses according to DSM-IV among people with ID compared with those without. Also, Cooper et al. (2015) found that the prevalence of psychiatric diagnoses according to the READ code were twice as high among people with
ID as among those without ID. The effect found in the present study is slightly larger than that found in these studies. This discrepancy may be explained by the differences in age distributions in the three studies, as old age, at least up until 75 years, has been found to be associated with higher rates of psychiatric morbidity among people with ID (Cooper, 1997b; Cooper et al. 2015). Differences in diagnostic criteria are also likely to account for some of the discrepancy, as is differences in the outcome measures (e.g. prevalence $v$. incidence).

The percentage of people with ID who had at least one psychiatric diagnosis during the study period decreased with age. Even though life expectancy is increasing among people with ID, it is still shorter than in the general population. Thus, the decrease in diagnoses among the oldest in the ID cohort may, at least partly, be due to a survival bias, i.e. that the oldest age group is healthier simply because those who had severe disorders would not have been included in the present study (as being alive was an inclusion criteria).

The largest OR for people with ID compared with those in the general population in the present study was found for diagnoses of psychotic disorders. This is well in line with previous studies investigating people with and without ID, where a considerable increase in overall psychotic disorders and schizophrenia specifically has been found for people with ID, regardless of which diagnostic system was used (Deb et al. 2001; Cooper et al. 2007a, 2015; Gentile et al. 2014; Howlett et al. 2015; Carey et al. 2016).

The $74 \%$ increased odds for diagnoses of affective disorders among people with ID in the present study is similar to what has been found in other community- 
based populations (Deb et al. 2001) and administratively defined cohorts (Howlett et al. 2015). However, Carey et al. (2016) found an almost sevenfold increased prevalence of affective disorders among people with ID in comparison with the general population when studying data from primary care. The different results may in part be explained by the use of different diagnostic systems or different outcome measures. However, it may also by that although people with ID may have increased risk of affective disorders, they are not likely to receive specialist care for this type of psychiatric diagnosis.

We found diagnoses of anxiety disorders to be more common among people with ID than in the general population, which is in line with some (Gentile et al. 2014; Carey et al. 2016), but not all (Deb et al. 2001; Hermans et al. 2013; Howlett et al. 2015), previous studies. One explanation to the different results found may be the use of different diagnoses systems or outcome measures. However, there are also other factors that may account for at least part of the discrepancy. The rate of anxiety disorders increases with age among people with ID (Cooper, 1997a; Hermans et al. 2013), but decreases with age in the general population (Ramsawh et al. 2009; Blay \& Marinho, 2012). Thus, the ratio of anxiety disorders among people with ID and the general population should increase with age. This could explain the discrepancy in results between the present study and that by Deb et al. (2001) and Howlett et al. (2015), as the people in these studies were younger than those in the present. The study population in (Hermans et al. (2013) had, however, a similar age distribution as our study group. A drawback of that study was the use of a standardised interview not adapted to people with ID, which may underestimate the true prevalence of anxiety disorders in the group of people with ID.

Although several studies have investigated personality disorders among criminal offenders with ID, not much research has been published on this dual diagnosis in a more general ID population. The limited evidence available suggests that people with ID are at a greater risk for diagnoses of personality disorders (Pridding \& Procter, 2008; Howlett et al. 2015). The increase in diagnoses of personality disorders in the present study is thereby in agreement with and adds important information to the knowledge base regarding personality disorders among people with ID.

People with ID seem to be more sensitive than the general population to developing a substance-abuse-related disorder (McGillicuddy \& Blane, 1999; Chapman \& Wu, 2012; van Duijvenbode et al. 2015). In the present study, only two percent of those with ID had a diagnosis of alcohol/substance-abuse-related disorders. This is at the low end of previously published results (Cooper et al. 2015; van Duijvenbode et al. 2015). As substance abuse is not necessarily a chronic state, the 11-year prevalence would be expected to be higher than point prevalences. Differences in the definition and selection of ID-group, study design, age groups studied, living conditions, severity of ID and definition of substance use may contribute to the variation in numbers. We found an almost threefold increase in odds of dementia diagnosis associated with having ID. Cooper et al. (2015) and Carey et al. (2016) both used primary care data to identify people with ID as well as diagnosis of dementia according to READ code. In both studies, about a fourth of the group of people with ID were aged 55 years, or older, and both studies found a large increase in risk of dementia among those with ID compared with the general population. However, Gentile et al. (2014) who let a psychiatrist diagnose all participants, found lower prevalence of dementia among people with ID compared with the population prevalence when using data from outpatient clinics. As the authors do not provide the age of the participants, age differences may be a possible explanation for the discrepancy with other studies. Another explanation may be that people with ID and dementia are more often treated in primary care, or that different diagnostic systems identify dementia differently in people with ID.

In the present study, $A D H D$ was the least frequent psychiatric diagnosis among people with ID, with less than one percent of the cohort getting a diagnosis during the study period. As people in this age group are unlikely to 'lose' their ADHD diagnosis, this number may be used as a prevalence estimate. Compared with other studies, it is a low one (Fox \& Wade, 1998; La Malfa et al. 2008). This may partly be explained by differences in age distributions, and that primary care is not included in the present study. However, it cannot be ruled out that ADHD is underdiagnosed among older people with ID in Sweden.

The co-existence of ID and psychiatric disorders does not only have a negative impact on the individual, but also places a burden on the health care system and family members. Therefore, further research into the understanding of diagnosis and treatment of such disorders in this vulnerable group of people is vital.

\section{Acknowledgements}

We would like to acknowledge the cooperation of the FUB, The Swedish National Association for People with Intellectual Disability.

\section{Financial Support}

This work was funded by Forte, the Swedish Research Council for Health, Working Life and Welfare no. 2014-4753. 


\section{Conflict of Interest}

None.

\section{Ethical Standards}

The authors assert that all procedures contributing to this work comply with the ethical standards of the relevant national and institutional committees on human experimentation and with the Helsinki Declaration of 1975, as revised in 2008. Ethical approval was obtained from the Regional Ethical Review Board in Lund (diary no 2013/15). The National Board of Health and Welfare as well as Statistics Sweden performed separate secrecy reviews in 2014 before providing access to the data. Analyses were performed using anonymised datasets.

\section{Availability of Data and Materials}

The data in the present study contain sensitive information on a very vulnerable group, i.e. people with ID. Even though the data are anonymised, it contains enough details to enable identification of single individuals. Therefore, in order to approve the study, the Regional Ethical Review Board in Lund made considerable restrictions regarding access to the data. This means we will not be able to provide other researchers with our data. However, as our database is compiled by register data only, other researchers may contact Statistics Sweden and the Swedish National Board of Health and Welfare to get access to the different registers included, and thereby recreate the database.

\section{References}

Alexander R, Cooray S (2003). Diagnosis of personality disorders in learning disability. British Journal of Psychiatry Supplement 44, S28-S31.re

Antonacci DJ, Attiah N (2008). Diagnosis and treatment of mood disorders in adults with developmental disabilities. Psychiatric Quarterly 79, 171-192.

Axmon A, Karlsson B, Ahlström G (2016). Health care utilisation among older persons with intellectual disability and dementia: a registry study. Journal of Intellectual Disability Research 60, 1165-1177.

Bakken TL, Helverschou SB, Eilertsen DE, Heggelund T, Myrbakk E, Martinsen H (2010). Psychiatric disorders in adolescents and adults with autism and intellectual disability: a representative study in one county in Norway. Research in Developmental Disability 31, 1669-1677.

Bhaumik S, Tyrer FC, McGrother C, Ganghadaran SK (2008). Psychiatric service use and psychiatric disorders in adults with intellectual disability. Journal of Intellectual Disability Research 52, 986-995.
Blay SL, Marinho V (2012). Anxiety disorders in old age. Current Opinion in Psychiatry 25, 462-467.

Bouras N, Martin G, Leese M, Vanstraelen M, Holt G, Thomas C, Hindler C, Boardman J (2004). Schizophrenia-spectrum psychoses in people with and without intellectual disability. Journal of Intellectual Disability Research 48, 548-555.

Carey IM, Shah SM, Hosking FJ, DeWilde S, Harris T, Beighton C, Cook DG (2016). Health characteristics and consultation patterns of people with intellectual disability: a cross-sectional database study in English general practice. British Journal of General Practice 66, 264-270.

Chapman SLC, Wu LT (2012). Substance abuse among individuals with intellectual disabilities. Research in Developmental Disability 33, 1147-1156.

Cheniaux E, Landeira-Fernandez J, Versiani M (2009). The diagnoses of schizophrenia, schizoaffective disorder, bipolar disorder and unipolar depression: interrater reliability and congruence between DSM-IV and ICD-10. Psychopathology 42, 293-298.

Cooper SA (1997a). Epidemiology of psychiatric disorders in elderly compared with younger adults with learning disabilities. British Journal of Psychiatry 170, 375-380.

Cooper SA (1997b). Psychiatry of elderly compared to younger adults with intellectual disabilities. Journal of Applied Research in Intellectual Disabilities 10, 303-311.

Cooper SA (1999). The relationship between psychiatric and physical health in elderly people with intellectual disability. Journal of Intellectual Disability Research 43(Pt 1), 54-60.

Cooper SA, Smiley E, Morrison J, Allan L, Williamson A, Finlayson J, Jackson A, Mantry D (2007a). Psychosis and adults with intellectual disabilities. Prevalence, incidence, and related factors. Social Psychiatry and Psychiatric Epidemiology 42, 530-536.

Cooper SA, Smiley E, Morrison J, Williamson A, Allan L (2007b). Mental ill-health in adults with intellectual disabilities: prevalence and associated factors. British Journal of Psychiatry 190, 27-35.

Cooper SA, McLean G, Guthrie B, McConnachie A, Mercer S, Sullivan F, Morrison J (2015). Multiple physical and mental health comorbidity in adults with intellectual disabilities: population-based cross-sectional analysis. BMC Family Practice 16, 110.

Coppus AM (2013). People with intellectual disability: what do we know about adulthood and life expectancy? Developmental Disabilities Research Reviews 18, 6-16.

Deb S, Thomas M, Bright C (2001). Mental disorder in adults with intellectual disability. I: prevalence of functional psychiatric illness among a community-based population aged between 16 and 64 years. Journal of Intellectual Disability Research 45, 495-505.

Deb S, Hare M, Prior L, Bhaumik S (2007). Dementia screening questionnaire for individuals with intellectual disabilities. British Journal of Psychiatry 190, 440-444.

Dieckmann F, Giovis C, Offergeld J (2015). The life expectancy of people with intellectual disabilities in Germany. Journal of Applied Research in Intellectual Disabilities 28, 373-382. 
Erkinjuntti T, Ostbye T, Steenhuis R, Hachinski V (1997). The effect of different diagnostic criteria on the prevalence of dementia. New England Journal of Medicine 337, 1667-1674.

Evenhuis HM, Hermans H, Hilgenkamp TI, Bastiaanse LP, Echteld MA (2012). Frailty and disability in older adults with intellectual disabilities: results from the healthy ageing and intellectual disability study. Journal of the American Geriatrics Society 60, 934-938.

Fox RA, Wade EJ (1998). Attention deficit hyperactivity disorder among adults with severe and profound mental retardation. Research in Developmental Disability 19, 275-280.

Gentile JP, Gillig PM, Stinson K, Jensen J (2014). Toward impacting medical and psychiatric comorbidities in persons with intellectual/developmental disabilities: an initial prospective analysis. Innovations in Clinical Neuroscience 11, 22-26.

Goldberg B, Gitta MZ, Puddephatt A (1995). Personality and trait disturbances in an adult mental retardation population: significance for psychiatric management. Journal of Intellectual Disability Research 39, 284-294.

Haveman M (2004). Disease epidemiology and aging people with intellectual disabilities. Journal of Policy and Practice in Intellectual Disabilities 1, 16-23.

Havercamp SM, Scott HM (2015). National health surveillance of adults with disabilities, adults with intellectual and developmental disabilities, and adults with no disabilities. Disability and Health Journal 8, 165-172.

Hermans H, Evenhuis HM (2010). Characteristics of instruments screening for depression in adults with intellectual disabilities: systematic review. Research in Developmental Disability 31, 1109-1120.

Hermans H, Beekman AT, Evenhuis HM (2013). Prevalence of depression and anxiety in older users of formal Dutch intellectual disability services. Journal of Affective Disorders 144, 94-100.

Howlett S, Florio T, Xu H, Trollor J (2015). Ambulatory mental health data demonstrates the high needs of people with an intellectual disability: results from the New South Wales intellectual disability and mental health data linkage project. The Australian and New Zealand Journal of Psychiatry 49, 137-144.

La Malfa G, Lassi S, Bertelli M, Pallanti S, Albertini G (2008). Detecting attention-deficit/hyperactivity disorder (ADHD) in adults with intellectual disability The use of Conners' Adult ADHD Rating Scales (CAARS). Research in Developmental Disability 29, 158-164.

Lidher J, Martin DM, Jayaprakash MS, Roy A (2005). Personality disorders in people with learning disabilities: follow-up of a community survey. Journal of Intellectual Disability Research 49, 845-851.

Mantry D, Cooper SA, Smiley E, Morrison J, Allan L, Williamson A, Finlayson J, Jackson A (2008). The prevalence and incidence of mental ill-health in adults with Down syndrome. Journal of Intellectual Disability Research 52, 141-155.

McGillicuddy NB, Blane HT (1999). Substance use in individuals with mental retardation. Addictive Behaviors 24, 869-878.
Melville CA, Cooper SA, Morrison J, Smiley E, Allan L, Jackson A, Finlayson J, Mantry D (2008). The prevalence and incidence of mental ill-health in adults with autism and intellectual disabilities. Journal of Autism and Developmental Disorders 38, 1676-1688.

Mindham J, Espie CA (2003). Glasgow Anxiety Scale for people with an intellectual disability (GAS-ID): development and psychometric properties of a new measure for use with people with mild intellectual disability. Journal of Intellectual Disability Research 47, 22-30.

Moreland J, Hendy S, Brown F (2008). The validity of a personality disorder diagnosis for people with an intellectual disability. Journal of Applied Research in Intellectual Disabilities 21, 219-226.

Moss S, Patel P (1997). Dementia in older people with intellectual disability: symptoms of physical and mental illness, and levels of adaptive behaviour. Journal of Intellectual Disability Research 41(Pt 1), 60-69.

Nettelbladt P, Goth M, Bogren M, Mattisson C (2009). Risk of mental disorders in subjects with intellectual disability in the Lundby cohort 1947-97. Nordic Journal of Psychiatry 63, 316-321.

Nilsson J, Ostling S, Waern M, Karlsson B, Sigstrom R, Guo X, Skoog I (2012). The 1-month prevalence of generalized anxiety disorder according to DSM-IV, DSM-V, and ICD-10 among nondemented 75-year-olds in Gothenburg, Sweden. American Journal of Geriatric Psychiatry 20, 963-972.

Ouellette-Kuntz H, Garcin N, Lewis MES, Minnes P, Martin C, Holden JJA (2005). Addressing health disparities through promoting equity for individuals with intellectual disability. Canadian Journal of Public Health 96, S8-S22.

Perez-Achiaga N, Nelson S, Hassiotis A (2009). Instruments for the detection of depressive symptoms in people with intellectual disabilities: a systematic review. Journal of Intellectual Disabilities 13, 55-76.

Pridding A, Procter NG (2008). A systematic review of personality disorder amongst people with intellectual disability with implications for the mental health nurse practitioner. Journal of Clinical Nursing 17, 2811-2819.

Ramsawh HJ, Raffa SD, Edelen MO, Rende R, Keller MB (2009). Anxiety in middle adulthood: effects of age and time on the 14-year course of panic disorder, social phobia and generalized anxiety disorder. Psychological Medicine 39, 615-624.

Reiss S, Szyszko J (1983). Diagnostic overshadowing and professional experience with mentally-retarded persons. American Journal of Mental Deficiency 87, 396-402.

Salvador-Carulla L, Rodriguez-Blazquez C, de Molina MR, Perez-Marin J, Velazquez R (2000). Hidden psychiatric morbidity in a vocational programme for people with intellectual disability. Journal of Intellectual Disability Research 44, 147-154.

Schutzwohl M, Koch A, Koslowski N, Puschner B, Voss E, Salize HJ, Pfennig A, Vogel A (2016). Mental illness, problem behaviour, needs and service use in adults with intellectual disability. Social Psychiatry and Psychiatric Epidemiology 51, 767-776.

SFS 1993:387 (1993). Act Concerning Support and Service for Persons with Certain Functional Impairments (In Swedish: 
Lag om stöd och service till vissa funktionshindrade (LSS)). Stockholm, Sweden.

Strydom A, Livingston G, King M, Hassiotis A (2008). Prevalence of dementia in intellectual disability using different diagnostic criteria. Journal of Policy and Practice in Intellectual Disabilities 5, 30-31.

van Duijvenbode N, VanderNagel JEL, Didden R, Engels RCME, Buitelaar JK, Kiewik M, de Jong CAJ

(2015). Substance use disorders in individuals with mild to borderline intellectual disability: current status and future directions. Research in Developmental Disability 38, 319-328.

WHO (2000). Healthy Ageing: Adults with Intellectual Disabilities: Summative Report. WHO: Geneva.

Yoo JH, Valdovinos MG, Schroeder SR (2012).

The epidemiology of psychopathology in people with intellectual disability: a forty-year review. International Review of Research in Developmental Disabilities 42, 31-56. 